\title{
Scaling of the turbulence transition threshold in a pipe
}

\author{
B. Hof, A. Juel, and T. Mullin \\ Manchester Center for Nonlinear Dynamics, \\ University of Manchester, Oxford Road, \\ Manchester, M13 9PL, UK
}

(Dated: December 4, 2018)

\begin{abstract}
We report the results of an experimental investigation of the transition to turbulence in a pipe over approximately an order of magnitude range in Re. A novel scaling law is uncovered using a systematic experimental procedure which permits contact to be made with modern theoretical thinking. The principal result we uncover is a scaling law which indicates that the amplitude of perturbation required to cause transition scales as $O\left(R e^{-1}\right)$.

PACS numbers: 47.20.-k,47.27.-i,47.60.+i

Keywords: Pipe flow, stability, transition, turbulence.
\end{abstract}

The puzzle of why the flow of a fluid along a pipe is typically observed to change from laminar to turbulent as the flow rate is increased has been the outstanding challenge of hydrodynamic stability for more than a century. The issue is both of deep scientific and engineering interest since most pipe flows are turbulent in practice even at modest flow rates. All theoretical work indicates that the flow is linearly stable [1], i.e infinitessimal disturbances added to the field will decay and disappear as they travel along the pipe and the flow will remain laminar. It is natural to assume that finite amplitude perturbations are therefore responsible for triggering turbulence and these become more important as the non-dimensionalized flow rate, the Reynolds number, $R e$, increases [2]. A question which may be asked is, if $\epsilon=\epsilon(R e)$ denotes the minimal amplitude of all finite perturbations that can trigger transition, and if $\epsilon$ scales with $R e$ according to

$$
\epsilon=O\left(R e^{\gamma}\right)
$$

as $R e \rightarrow \infty$, then what is the exponent $\gamma$ [11]? A negative value of $\gamma$ is anticipated and one substantially less than zero would indicate that the sensitivity of the laminar flow increases rapidly with $R e$, i.e. the basin of attraction of the laminar fixed point diminishes rapidly as $R e$ increases. Current estimates for $\gamma$ suggest that for shear flows it lies within the range $-1 \geq \gamma \geq-7 / 4$ from various model studies [17, 19] and numerical simulations [21] where and exponent strictly less than -1 is required for transient growth 12. A significant challenge is to relate this theoretical concept to observation in a quantitative manner although some limited data is available [6]. In this Letter we provide evidence from a novel experiment which suggests a way forward and provides striking evidence for an exponent of -1 which points to a generic transition 12.

The stability of Hagen-Poiseuille flow in a long circular pipe has intrigued scientists for more than a century since Reynolds' 3] experimental investigations. Reynolds showed that when the parameter we now call the Reynolds number Re was greater than approximately
2,000 then turbulent flow became the norm in practice. $R e$ is usually defined as $R e=U d / \nu$ where $U$ is the mean speed of the flow, $d$ is the diameter of the pipe and $\nu$ is the kinematic viscosity of the fluid. Importantly, he also showed that if the inlet disturbances to the pipe are minimized then laminar flow can be maintained to higher flow rates than if they are not. This finding has been extended in modern times to Reynolds numbers of $\approx 100,000$ [4] in transient flows by taking extraordinary care.

The process whereby turbulence arises is not understood either in outline or in detail and this problem is an unresolved scientific challenge. Any advance towards an understanding of the fundamentals involved will have widespread impact on flows of practical interest. For example, the flows in oil and gas pipelines are often run inefficiently turbulent to avoid the large pressure fluctuations of the transitional regime. Moreover, the control of turbulence is a dream of many practitioners, just as an understanding of turbulence is the desire of many scientists.

In general terms, pipe flow may be considered as a nonlinear dynamical system $d \mathbf{u} / d t=f(\mathbf{u}, R e)$ which represent the Navier Stokes equations subject to appropriate forcing and boundary conditions. The single parameter $R e$, determines the dynamical state of the system such that there is one linearly stable fixed point for all $R e$ and another possible attractor, turbulence, when $R e>R e_{c}$. Hence when $R e<R e_{c}$ all initial conditions are attracted to the laminar state which is the global attractor for the system. When $R e>R e_{c}$ nearly all initial conditions give rise to turbulence so that the laminar state is now a local attractor. In practice, $R e_{c} \approx 2000$ so that all disturbances will decay as $t \rightarrow \infty$ for values of $R e$ smaller than this. These arguments are consistent both with Reynolds" original observations and modern experimental results [5, 6, 7, 8]. Almost all experimental studies of the problem have been concerned with pressure gradient driven flows so that large fluctuations in flow rate and hence $R e$ can, in principle, occur upon transition. In one exception to this [8] a constant mass flux system is used 




FIG. 1: Schematic of the 'long pipe' experimental system.

where the flow is pulled by a piston and this accurately fixes Re. Impulsive perturbations are used to produce a finite amplitude stability curve such that disturbances with amplitudes greater than a threshold produce turbulence while smaller ones decay downstream. The results are consistent with those obtained with pressure driven systems [5, 6, 7] so that localized 'puffs' and 'slugs' are found at low Re and fully developed turbulence at larger flow rates.

Modern theoretical research may be broadly split into two approaches. In one, initially small disturbances on the laminar state grow in a transient phase [1, 18] until they reach a sufficiently large amplitude that nonlinear effects become important. These ideas have been explored for various low-dimensional models [9] and applied to plane Poiseuille flow [10] and scaling laws for the amplitude of the perturbation as a function of $R e$ have been provided. An alternative point of view 13] is that the turbulent state originates from instabilities of a finite amplitude solution which is disconnected from the base state. The basin of attraction of the turbulent state grows with $R e$ so that any small perturbation will kick the laminar solution towards it. Such solutions of the Navier Stokes equations are known to exist in other flows 14, 15, 16] but their existence has not yet been shown in pipe flows.

In drawing a connection between experimental observations and predictions from models the concept of a perturbation needs to be defined. In models, the temporal and spatial form of the perturbation can be accurately specified. On the other hand, experimentalists rely on injecting and/or subtracting fluid through slits or holes in an attempt to mimic the mathematical process. The perturbation can be either periodic [6, 7] or impulsive [ 8] but specifying an essential measure such as the scale of the amplitude is difficult. Indeed, resolving the pertinent part of the physical perturbation which gives rise to transition is in itself a difficult exercise although progress is being made in that direction [7]. In an attempt to address this issue we have devised a novel type of perturbation which permits a scaling analysis and thereby allows a closer connection to be made with theory.

The experimental system can be regarded as a large computer controlled syringe which has the capability of pulling water at a fixed mass flux along a precision bore tube. We have two such experimental facilities in our laboratory and we will only outline details of the new rig as the first system has been described previously [8]. The pipe consisted of a $20 \mathrm{~mm}$ diameter Perspex tube which was $15.7 \mathrm{~m}$ in length and constructed from 105 machined sections each of which was $150 \mathrm{~mm}$ long. The sections were fitted together and aligned with a laser on a steel base. Still water was drawn from a tank through a trumpet shaped inlet into the tube and a similar expansion connected the $260 \mathrm{~mm}$ diameter piston to the tube. The flow state was monitored using flow visualization and recorded at various spatial locations using video cameras whose images were stored for further processing. Laminar flow was achieved for $R e \leq 24,000$ verifying the quality of the construction. We will only report results for $R e \leq 18,000$ and the flow was fully developed at the disturbance injection point up to $R e=16,000$. A schematic diagram showing the experimental arrangement for the 'long pipe' is given in Fig. 11 and the shorter pipe was constructed in a very similar way but was 150 diameters long.

The stability of the flow was probed using a perturbation which was applied a sufficient number of diameters from the inlet to ensure fully developed flow over the $R e$ ranges investigated in the two systems. This distance was 75 and 530 pipe diameters for the short and long pipes respectively. A single boxcar pulse of fluid was injected tangentially into the flow via a ring of six equally spaced $0.5 \mathrm{~mm}$ holes to provide the disturbance. The injection system contained two high speed solenoid valves with switching times of $\approx 1 \mathrm{~ms}$ and the rise and fall times of the perturbation were limited by the inertia of the piston. The quantities of fluid injected were in the range 0.01 to $0.1 \%$ of the total volume flux where the larger values were required to cause transition at smaller $R e$. 
This novel injection system enabled us to vary the duration and amplitude of the perturbation independently. We show in Fig.2] a typical pressure trace of a perturbation of magnitude $\Delta p$ and width $\Delta t$. It can be seen that a reasonable approximation to a box-car function is achieved with relatively small amplitude ringing at the switching times. The pressure was measured at a single location to illustrate the form of the perturbation and measurement of pressure gradient in this format remains a technical challenge. Hence we use the displaced volume flux $\Phi_{i n j}$ from the injector in our definition of the amplitude of the perturbation since this sets the spatial extent of the disturbed flow and defining the amplitude in terms of relative volume fluxes enables a direct connection with theory. In principle, the perturbation will have a global effect on the flow field but checks using injection and suction [8] show that it is localized in practice.

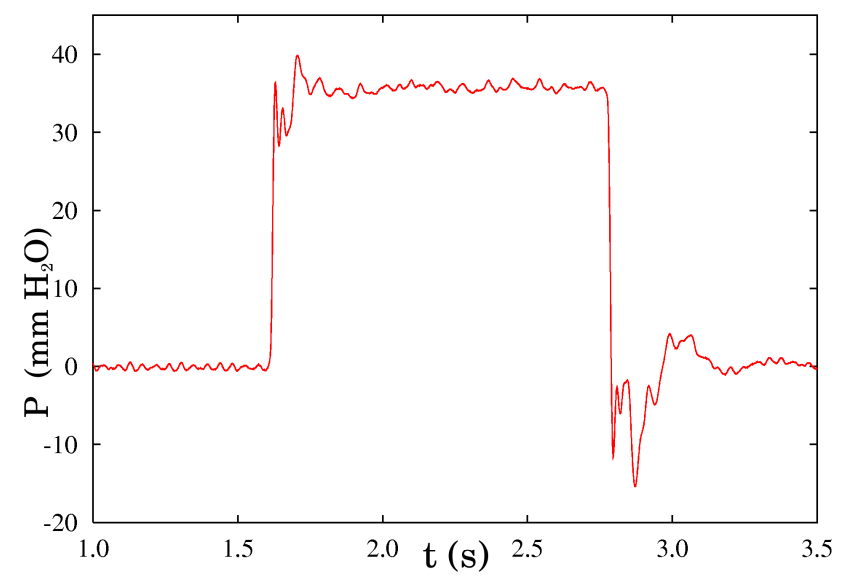

FIG. 2: A typical pressure time series for a perturbation of amplitude $\Delta p=37 \pm 1 \mathrm{~mm} \mathrm{H}_{2} \mathrm{O}$ and width $\Delta t=1.2 \pm 0.01 \mathrm{~s}$.

As discussed above, this means of injecting a disturbance permits the amplitude and duration of the perturbation to be varied independently. In Fig. [3] we show stability curves for two different values of $R e$ where the amplitude of perturbation required to cause transition is plotted as a function of the length of the perturbed flow in pipe diameters. This scale was set by injecting for a prescribed time and, since the disturbed flow was advected at the mean speed of the pipe flow, an estimate of the spatial extent of the perturbation immediately downstream of the injection point was made. This is denoted by length* in Fig. [3 and use of this scaling collapses the two sets of data onto a single curve.

Perturbations with amplitudes such that they were below the curve did not cause transition and hence decayed as they propagated downstream. On the other hand, disturbances which had amplitudes above the curve gave rise to sustained disordered flow downstream which had the form of a localized 'puff' [5] at $R e=2170$ or a patch of turbulence at $R e=4000$. As discussed elsewhere [8], the threshold is probabilistic so that a mean value can be estimated with a narrow well-defined width. These are denoted by the error bars in Fig. [3 which indicate the width of the experimentally determined probability distribution of the transition obtained from forty rehearsals of the experiment.

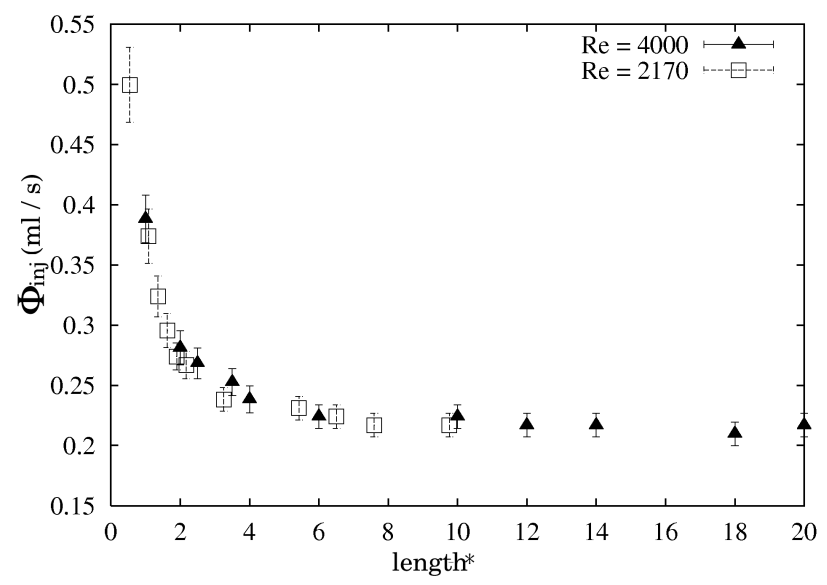

FIG. 3: Stability curves measured for $R e=2170$ and $R e=$ 4000. Each data point was obtained from forty runs of the experiment and the error bars correspond to the widths of the determined probability distributions [8]. The abscissa is the 'length"' of the initially disturbed flow in pipe diameters.

The data in Fig. [3] also shows that the amplitude of perturbation required for transition is independent of its length when more than six pipe diameters are initially disturbed. Shorter length perturbations result in a nonlinear response and these results are in accord with previous work [8] where a short triangular form perturbation was used.

We reinforce the above scaling argument with the results shown in Fig. 4 which contain data in the range $2,000 \leq R e \leq 5,500$ where $95 \%$ fully developed flow was achieved with the shorter pipe. Three stability curves are presented and these will now be discussed in turn. In the first, a short, $0.2 \mathrm{~s}$., duration pulse was used and this corresponds to a disturbance length of one pipe diameter at $R e=2,000$. This exhibits nonlinear behavior such that a rapidly increasing perturbation amplitude is required to cause transition as $R e=2,000$ is approached. The data are in agreement with those obtained in previous experiments $[8]$. In the next, a long duration (1.8s.) pulse was used and this corresponds to perturbing nine pipe diameters at $R e=2,000$. Here, the independence of the amplitude of perturbation required to cause transition discussed in connection with Fig. [3 for lengths $\geq 6 D$ is confirmed. In this case, independence has been uncovered over a range of $R e$. The final data set was obtained by varying duration of the pulse in proportion to the mean flow such that the length of the flow field which was initially disturbed was kept constant at 2.25 pipe 


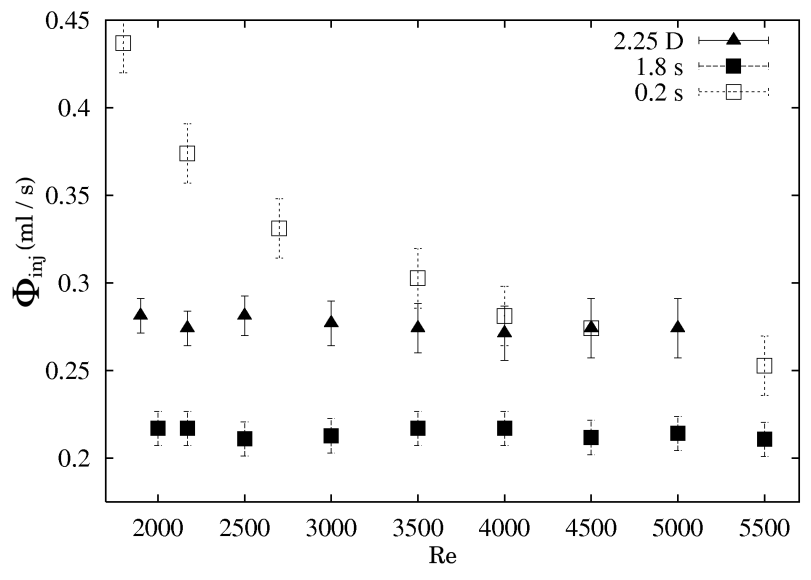

FIG. 4: Stability curves measured in the 'short' pipe in the range $2,000 \leq R e \leq 5,500$. Fixed duration perturbations were used to obtain the loci labelled $0.2 \mathrm{~s}$. and $1.8 \mathrm{~s}$ respectively. The data set labelled $2.25 D$ were measured using a variable duration pulse such that 2.25 diameters of the pipe flow were perturbed.

diameters. This corresponds to a perturbation of width $0.45 \mathrm{~s}$. at $R e=2,000$. The data reinforces that scaling of the perturbation by the mean flow is valid. An alternative way of viewing this data set is to consider it as a sampled set from a horizontal cut taken through a family of parallel stability curves, one for each value of the $R e$ set in the experiment.



FIG. 5: A log-log plot of the stability curve obtained using the long pipe. The range of $R e$ covered is 2000 to 18,000 and the amplitude of the perturbation has been non-dimensionalized by the respective mass flux in the pipe. The least squares fitted line has a slope of -1 as indicated.

It is clear that the two level thresholds cannot continue much below $R e=2,000$ since experimental evidence suggests that turbulent flow cannot be maintained below this value $[5,8]$. It is equally unlikely that the locus will sim- ply come to an end in parameter space. In this region, we observe the transient growth of puffs which can persist for many tens of pipe diameters. This interesting behavior will take considerable experimental effort to resolve and is the subject of an ongoing investigation.

An appropriate scaling of the amplitude of the perturbation is the relative mass flux of the perturbation to that in the pipe. Clearly, doing this for the two horizontal loci in Fig. 4 will produce a proportionality of the form $O\left(R e^{-1}\right)$. We next present results from the long pipe in Fig. [5 where we were able to test this finding over an order of magnitude range of $R e$. Here we used a perturbation of $1.8 \mathrm{~s}$ duration and find the same $O\left(R e^{-1}\right)$ scaling.

Our investigation has shown that the amplitude required for transition in a pipe is constant for a sufficiently long perturbation. Shorter perturbations show nonlinear dependence but, these may be scaled with $R e$ so that the amplitude of perturbation required for transition has a $R e^{-1}$ dependence. This is agreement with recent theoretical estimates for asymptotically large $R e[22$ which indicates that the theory may apply over a surprisingly large range of $R e$. Hence we provide an advance towards forming a closer connection between modern theoretical approaches and experiment.

[1] P.G. Drazin \& W.H. Reid An Introduction to Hydrodynamic Stability Theory (1980) Cambridge University Press.

[2] Lord Kelvin (1887) Phil. Mag. 5,459.

[3] O. Reynolds (1883) Proc. Roy. Soc. A 35,84.

[4] W. Pfenniger (1961) InBoundary Layer and Flow Control,Pergamon (ed. G.V. Lachman),970.

[5] I. J. Wygnanski and F.H. Champagne (1973) J. Fluid Mech. 59,281.

[6] A. A. Draad, G. Kuiken and F.T.M. Nieuwstadt (1998) J. Fluid Mech. 377,267.

[7] G. Han, A. Tumin and I. Wygnanski (2000) J. Fluid Mech. 419,1.

[8] A. G. Darbyshire and T. Mullin (1995) J. Fluid Mech. $\mathbf{2 8 9}, 83$.

[9] J. S. Baggett and L. N. Trefethen (1997) Phys. Fluids $\mathbf{9}, 1043$.

[10] S.J. Chapman (2002) J. Fluid Mech. 451,35.

[11] L.N. Trefethen, A.E. Trefethen, S.C. Reddy, and T.A. Driscoll (1993) Science 261,578.

[12] F. Waleffe (1995) Phys. Fluids 7,3060.

[13] F. Waleffe (1997) Phys. Fluids 9,883.

[14] D. K. Anson, T. Mullin and K. A. Cliffe (1989) J. Fluid Mech. 207,475.

[15] D. R. Barnes and R. R. Kerswell (2000) J. Fluid Mech. 417,103.

[16] O. Dauchot and F. Daviaud (1995) Phys. Fluids 7,335.

[17] A. Mesguer (2003) Phys. Fluids 15,1203.

[18] S. Grossmann. (2000) Rev. Mod. Phys. 72,603.

[19] B. Eckhardt and A. Mersmann. (1999) Phys. Rev. E 60,509. 
[20] R.J. Leite (1959) J. Fluid Mech. 5,81.

[22] J. Chapman (Private communication).

[21] H. Shan, Z. Zhang and F. T. M. Nieuwstadt (1998) Int. J. Heat and Fluid Flow 19,320. 